\title{
AMAZÓNAS
}

Revista científica del Amazonas

Volumen 3 Número 5

Enero-junio 2020

\section{Percepción estudiantil de la calidad del servicio universitario: caso de una Universidad Ecuatoriana}

\author{
Student Perception of the quality of the University Service: Case of an Ecuadorian \\ University
}

Sarmiento Orna, Diana Alexandra ${ }^{12}$

ORCID: https://orcid.org/0000-0002-1807-7554

Vinueza Martínez, Jorge Luis ${ }^{13}$

ORCID: https://orcid.org/0000-0003-1966-43952

\section{Resumen}

El presente estudio tiene como objetivo mostrar la percepción de los estudiantes sobre la calidad del servicio universitario de una Institución de Educación Superior Ecuatoriana. La metodología de investigación aplicada es de tipo no experimental, seccional y descriptiva, mediante la aplicación de una encuesta de opinión que permitió conocer la percepción de una población aproximada de 3.802 estudiantes de pregrado y 180 de postgrado, respuestas codificadas en un corpus textual para su análisis, cuyos resultados permitieron conocer como criterios más frecuentes las palabras conocimiento $(\chi 2=192.6$, $\mathrm{p}<0.0001)$ y comodidad $(\chi 2=135.05, \mathrm{p}<0.0001)$ relacionadas a las dimensiones de calidad del servicio: elementos tangibles, fiabilidad, capacidad de respuesta, seguridad y empatía, que impulsan procesos de mejora para el desarrollo académico universitario público.

Palabras clave: Calidad, educación superior, percepción, servicio público.

\section{Abstract}

The purpose of this study is to show the students' perception of the quality of the university service of an Ecuadorian Higher Education Institution. The applied research methodology is of a non-experimental, sectional and descriptive type, through the application of an opinion survey that allowed to know the perception of an approximate population of 3,802 undergraduate and 180 postgraduate students, responses coded in a textual corpus for analysis, whose results allowed to know as more frequent criteria the words knowledge ( $22=192.6, \mathrm{p}<0.0001)$ and comfort $(\mathrm{c} 2=135.05, \mathrm{p}<0.0001)$ related to the dimensions of service quality: tangible elements, reliability, capacity of response,

\footnotetext{
${ }^{12}$ Universidad Estatal de Milagro, Milagro, Ecuador

${ }^{13}$ Universidad Estatal de Milagro, Milagro, Ecuador
} 


\section{AMAZƠNAS}

security and empathy, which drive improvement processes for public university academic development.

Key Words: Quality, higher education, perception, public service.

\section{Introducción}

En el contexto educativo nacional, desde el 2013 evoluciona un modelo que promueve políticas calidad en la evaluación institucional para las Instituciones de Educación Superior (IES), criterios como los presentados en el Modelo de Evaluación institucional de Universidades y Escuelas Politécnicas (2018), tales como la organización, recursos e infraestructura, claustro de profesores, estudiantes, vinculación con la sociedad e investigación (Consejo de Evaluación, 2018, p. 6-7), permiten alcanzar estándares de calidad, para una mejora continua y auto-reflexiva de sus actores que intervienen en el proceso educativo, esto mediante la construcción de una cultura participativa y de visión compartida (Asamblea Nacional del Ecuador, 2010, p.40).

Dadas las condiciones que anteceden, es necesario que las instituciones del sector público que ofertan la educación superior conozcan ¿En qué influye la evaluación de la gestión como soporte de los procesos sustantivos de la academia, reflejados en la calidad del servicio universitario?, donde el estudiante es el beneficiario principal. Según modelo de Cervantes (2018) basado en el triángulo del servicio orientado al cliente, los administradores de nivel superior, son los responsables de motivar, evaluar e implementar las estrategias necesarias a través de acciones correctivas sobre el portafolio de productos y servicios ofertado por la institución (Cervantes et al., 2018, p.40), mediante una correcta planificación, organización, dirección y control, y así introducir una imagen institucional innovadora que los distinga entre la competencia.

Es así, que el presente estudio toma el caso de la Universidad Estatal de Milagro (UNEMI), ubicada en la ciudad de Milagro, provincia del Guayas en Ecuador, como instancia de experimentación, tiene por objeto evaluar cualitativamente la percepción estudiantil sobre la calidad de los servicios ofertados en esta institución, cuyos resultados permitan a los administradores y servidores públicos que laboran en la institución, conocer, medir, analizar y revisar aspectos de mejoras a las políticas de gestión académica y administrativa sobre la brecha de valor detectada en cuanto a la satisfacción del servicio percibido por lo estudiantes.

\section{Marco Teórico}

La Organización Internacional de Normalización (ISO), define con "calidad" aquellas organizaciones que cuentan con un sistema activo y operante para administrar, renovar e innovar la calidad de sus servicios (Cervantes, Peralta, Salgado, \& Stefanell, 2018, p.31). Para Vergara, J. C. y Quesada V. (2011) calidad es la conformidad y satisfacción que percibe la comunidad sobre los servicios; concepto que ratifican Tumino \& Poitevin (2013), al mencionar que la calidad es subjetiva desde la percepción del usuario. 


\section{AmAZUNANAS}

En este sentido, para nuestro estudio, el término "servicio" es un concepto vinculado como la actividad o beneficio que brinda satisfacción al consumidor o sujeto beneficiario (Duque Oliva, 2005, p.64). Significa entonces que "calidad del servicio" es la impresión de satisfacción percibida por aquellos aspectos tangibles e intangibles, (Palominos, Quezada, Osorio, Torres, \& Lippi, 2016, p.132). Cristian Villalba Sanchez (2013) en su investigación sobre "La calidad del servicio", compila los modelos más representativos sobre la percepción y su aplicación en el contexto de las universidades, tales como: modelo americano, modelo nórdico, desarrollo estructural del concepto de calidad del servicio, y modelos aplicados en contextos ecuatorianos, según se aprecia en la Figura 1.

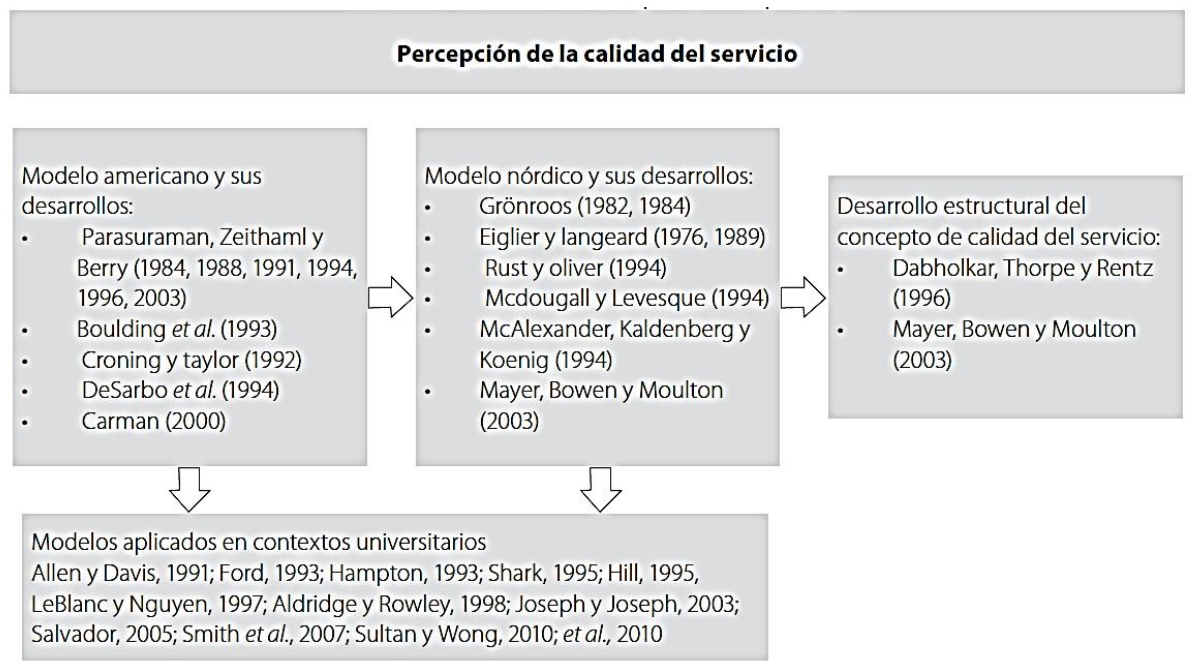

Figura 1. Tendencias de la percepción de la calidad del servicio.

Fuente: Tomado de (Sánchez, 2013, p.59)

Pero ¿Cómo podemos medir la calidad del servicio?. Para Feldman, el aplicar instrumentos de evaluación a través de preguntas abiertas, permiten conocer la percepción real de los estudiantes (Feldman, 2002, p.267), mediante la demostración de juicios o concepciones de la imagen institucional sobre el contexto del sistema educativo (D’Armas Regnault et al., 2018, p. 3), donde la postura estudiantil, según Suescún (2015), enriquece la crítica para mejorar el sistema educativo, mediante la aplicación de técnicas, metodologías y didácticas pedagógicas inclusive.

En el marco de las observaciones anteriores, expertos explican que entre los criterios que mejor se aplica a las universidades para conocer el nivel de calidad del servicio, se deben abarcar criterios a más de la interacción del aprendizaje entre profesores y estudiantes, evaluar las condiciones físicas o infraestructura institucional hasta aspectos interactivos de atención y servicio relacionado al buen trato del personal administrativo y de servicios (Vergara, J. C. y Quesada, 2011, p. 119). En efecto, estos criterios, se conectar con las dimensiones del conocido modelo Parasuraman, tales como: elementos tangibles, fiabilidad, capacidad de respuesta, seguridad y empatía (Alvarado-Lagunas, Luyando-Cuevas, \& Picazzo-Palencia, 2015, p.63), donde la escala Servqual y Servperf, son instrumentos diseñados para evaluar la calidad del servicio (Duque Oliva \& Chaparro Pinzón, 2017, p. 166), permitiendo identificar las expresiones significativas sobre la 


\section{AMAZONAS}

satisfacción, necesidad y expectativa percibida del estudiante sobre los centros de educación superior públicos (Villalba Sanchez, 2013, p.70), siendo ésta la voz la que retroalimenta y permite mejorar las políticas públicas e institucionales, según Espinosa, et al (2017).

Para Carretero Diaz (2004), la calidad es en un problema de percepción del usuario que se beneficia de un servicio, conocimiento que se traduce en el cerebro como un estímulo decodificado a través de los sentidos, proceso sensorio - cognitivo complejo que activa el conocimiento, y transforma los estímulos mediante una reorganización cerebral(Correa, Agila, José, \& Palacios, 2012, p. 128); dando lugar a una representación legítima de las expectativas e intereses de los usuarios (Iacovidou, Gibbs, \& Zopiatis, 2009, p. 148), sobre la valoración de juicio subjetiva de las sensaciones obtenidas (Ortega Carrasco, Veloso Toledo, \& Hansen, 2018, p.103). Sin embargo, esta percepción debe ser tratada con proyectos de mejora para la calidad total, con el compromiso principal del nivel jerárquico superior (Carretero Diaz, 2004, p.533).

Basado en planteamientos que anteceden, a nivel nacional se han realizado investigaciones, como la sostenida por Salazar Yépez \& Cabrera Vallejo (2016) en su trabajo "Diagnóstico de la Calidad de Servicio", realizado en la Universidad Nacional de Chimborazo, con instrumentos técnicos de recolección de información como el cuestionario Servqual, donde se evaluó las dimensiones objeto de la investigación, con resultados concretos acerca del estado de la calidad del servicio en la institución; siendo de mayor percepción los elementos tangibles, dado que transmiten comodidad según opinión.

En su investigación, Saltos Aguilar \& Arguello Erazo (2017), refieren que los indicadores de calidad son factores claves que determinan el éxito o fracaso del sistema de gestión educativa institucional; parámetros que se ajustan a las directrices del sistema educativo macro, que pueden ser locales, nacionales o internacionales (Roccaro, 2002, p.1). Para Alvarado Peña \& Díaz De León (2015), en estudio realizado "La calidad de servicio en universidades públicas de México" uno de sus resultados recae en la dimensión de empatía del personal en la gestión administrativa, donde los estudiantes refieren que el servicio es cordial en ocasiones, y en otras reciben un trato injusto e indiferente; como puede observarse, la supervivencia de las IES dependerá del constante compromiso y reflexión para innovar los modelos de calidad en la administración pública donde se evidencia el grado de respuesta de servidores públicos a las demandas del ciudadano - usuario.

Precisando de una vez, la percepción de la calidad de servicio permite elaborar un constructo teórico sobre la representación social de las IES, resultados que llevan a los niveles estratégicos a la reflexión sobre su gestión, contribución a la aplicación de la Constitución de la República del Ecuador (2008) para garantizar el acceso a educación gratuita hasta el tercer nivel, mediante la interculturalidad sin discriminación alguna (Asamblea Nacional del Ecuador, 2010, p.7), teniendo en cuenta aún existen nodos críticos por resolver y que se lograrán a través de la ruptura de modelos tradicionales (Aguilar-Gordón, 2018, p.28), en este propósito es necesario no olvidar que "La calidad es un término demasiado duro y excluyente en ocasiones, porque escoger supone excluir" (Carretero Diaz, 2004, p.557). 


\section{AMAZONAS}

\section{Metodología}

En este estudio se evaluó la percepción sobre la calidad del servicio universitario, con una investigación bajo la metodología cualitativa no experimental de tipo descriptiva, iniciando con el análisis de documentos de investigación con el desarrollo de tres fases: 1) identificar fuentes de información, 2) revisión de la literatura y 3) extracción y recopilación de información de las fuentes bibliográficas (Carrasco Proaño et al., 2018, p. 5), que sirvieron de referencia para elaboración del marco teórico, y que permitió argumentar las conclusiones y discusión del caso.

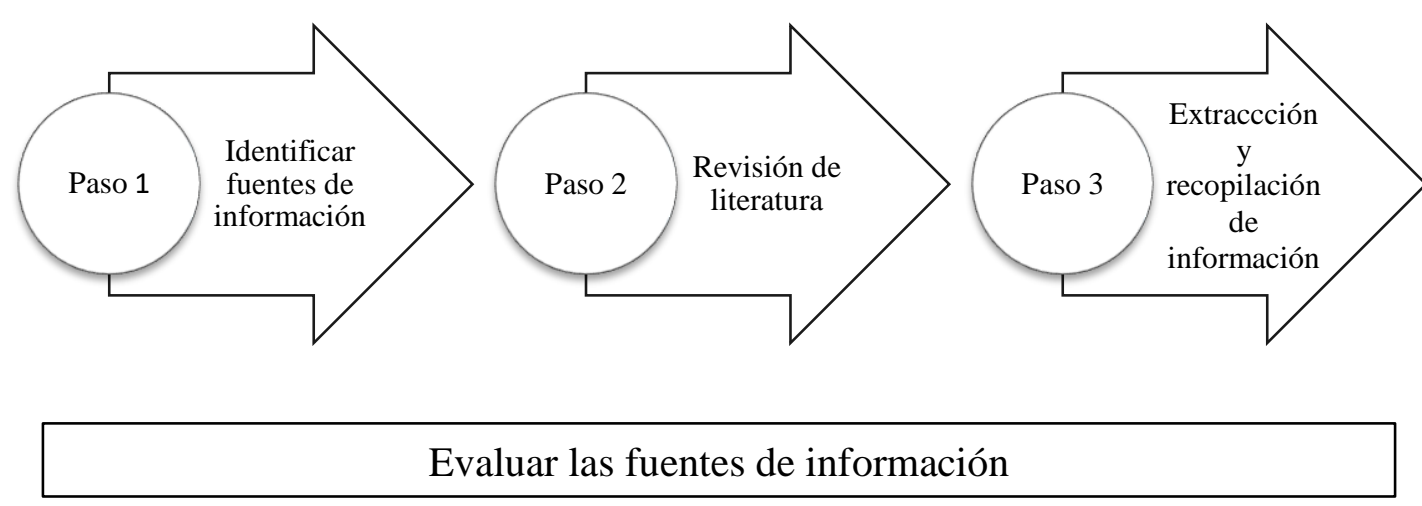

Figura 2. Fases para tratamiento de información.

Fuente: Elaboración propia de los autores (2019)

Donde, en la fase 1, se identificaron las variables para limitar la búsqueda de información, luego se realizó la búsqueda en las diversas fuentes de información catalogadas, documentos que fueron importados al gestor de contenidos de Mendeley, donde se construyó la base de datos para evaluar la información. En la etapa 2, de revisión de literatura, se exploró el aporte teórico de los autores para argumento de los constructos, y, por último, en la etapa 3 se extrajo la información más relevante sobre percepción de calidad de servicio para el desarrollo de la investigación.

Posterior, se procedió con la aplicación de análisis textual a través de la lexicometría, que considera la concurrencia de los criterios textuales y que redefine "expresiones conceptuales desde el punto de vista de su representación colectiva" (Romero-Pérez, Alarcón-Vásquez, \& García-Jimenez, 2018, p. 28), que organiza datos obtenidos de estudiantes de la Universidad Estatal de Milagro, en una encuesta de opinión con preguntas abiertas, relacionados a la problemática de la investigación, para comprender la percepción que existe sobre la calidad del servicio y que concluye por la representación de índices de coincidencias de palabras (repetidas o más usadas) agrupadas con el valor más alto de Chi cuadrado $(\chi 2)$. 


\section{AMAZÖNAS}

\section{Ámbito de la investigación}

El estudio se realizó en la Universidad Estatal de Milagro, ubicada en Ecuador, en la provincia del Guayas - Región 5, ciudad de Milagro, que al término del presente informe oferta 20 carreras presenciales de pregrado y 5 de postgrado, con sus ejes transversales de academia, investigación, vinculación y gestión, para el desarrollo de la sociedad; atiende aproximadamente a 4.534 estudiantes de pregrado y 555 de postgrado en modalidad presencial, matriculados hasta el primer semestre del 2018.

\section{Sujeto de la investigación}

El análisis fue conformado por los estudiantes como usuarios beneficiarios del servicio universitario público que brinda la Universidad Estatal de Milagro, en su campus, los mismos que forman parte de carreras de pregrado y postgrado. Véase Tabla 1.

\section{Tabla 1.}

Sujetos de la investigación.

\begin{tabular}{ll}
\hline Estudiantes & Cantidad \\
\hline Pregrado & 4.534 \\
Postgrado & 555 \\
\hline
\end{tabular}

Fuente: Informe de Gestión UNEMI (2018)

La Universidad Estatal de Milagro en busca de cubrir la brecha social con calidad y pertinencia académica, en la actualidad atiende una alta demanda de la ciudadanía de Milagro, llegando alcanzar desde el 2008 la cantidad de 3.567 estudiantes y al 2018 un número de 15.350 estudiantes; lo que representa un crecimiento de la población estudiantil; compromiso social que incrementa la tasa de acceso a la educación superior, para brindar una educación de calidad.

\section{Construcción del corpus}

Para la construcción del corpus se utilizó como instrumento una encuesta con preguntas abiertas, basado en investigación cualitativa no experimental de tipo descriptiva, en la que se introdujo cuestionamientos puntuales que motivan a los individuos exteriorizar su actitud y emoción (Ortega Márquez \& Márquez Fernández, 2017, p.49); dimensiones estructuradas de la siguiente forma: a) Pienso que los servicios que ofrece la UNEMI son..., b) Los servicios universitarios de la UNEMI me brindan..., y c) El servicio universitario mejoraría sí.

La selección de sujetos a encuestar fue al azar, aplicado a la población total de estudiantes regulares, consultados a través de la plataforma informática Sistema de Gestión Académica (SGA), alcanzando un total de 3.802 respuestas correspondientes a estudiantes de pregrado y 180 a estudiantes de postgrado, respuestas que fueron codificadas en un corpus textual para el respectivo tratamiento, procesamiento y análisis de datos. 


\section{Análisis del corpus}

En el análisis lexicométrico unidimensional y multidimensional se obtiene una nube de palabras frecuentes que representa el contexto del lenguaje que representa gráficamente las similitudes y asociaciones que existen entre las palabras claves (Romero-Pérez et al., 2018). Las respuestas fueron segmentadas en el corpus en base a las tres preguntas abiertas, referidas en el instrumento aplicado, realizándose el análisis en el software Iramuteq 0.7 alpha 2 , en interfaz de $\mathrm{R}$, que verifica las clases más representativas que están en los valores más altos del Chi-cuadrado (x2).

\section{Resultados}

La construcción del corpus textual dio paso al procesamiento de datos en el software aplicado, con el resultado de los datos cualitativos sobre el 19.52 de ocurrencias de palabras, agrupados en tres unidades de texto basadas en las preguntas; 2,848 número de formas de recuento de un texto, número de hápax en 1.038 (5.31\% de ocurrencias $50.58 \%$ de formas); siendo este el número de frecuencia de aparición de texto igual a uno (Rojo, 2017, p.124), y la media de ocurrencias por textos de 6.520,67.

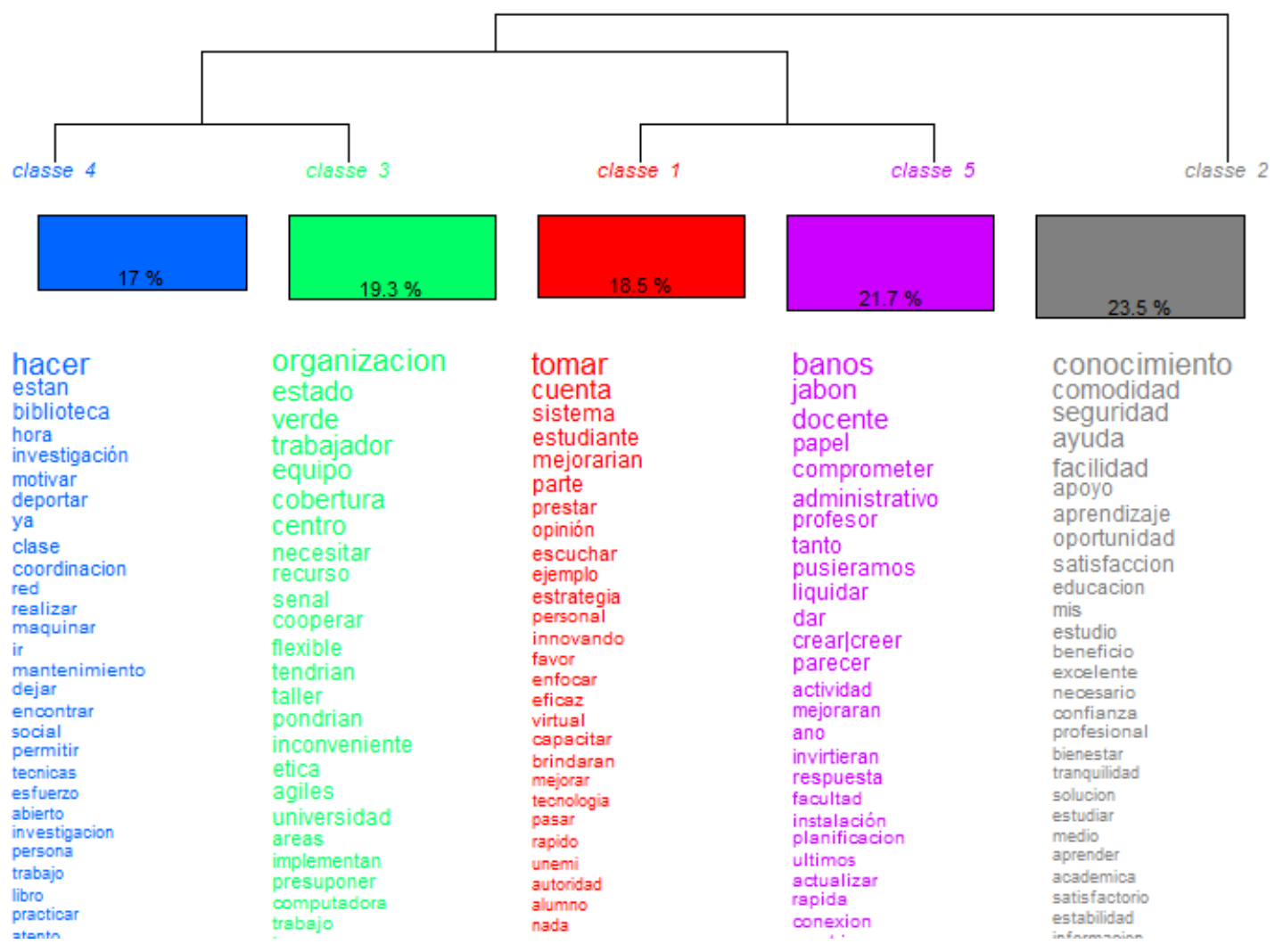

Figura 3. Dendrograma de Factores de percepción estudiantil de la calidad del servicio en UNEMI.

Fuente: Elaboración propia de los autores (2019). 
En la Figura 3 se muestran 5 clases o rangos de caracteres a las que puede corresponder una palabra (Rojo, 2017, p.132), según la siguiente clasificación o segmentación:

Tabla 2. Características de las clases ubicadas en el Dendrograma de Factores de percepción estudiantil de la calidad del servicio universitario en UNEMI.

\begin{tabular}{ll}
\hline Segmentación & Descripción \\
\hline Clase 1 & Compromiso y disposición para la atención. \\
Clase 2 & Riesgo sobre el servicio. \\
Clase 3 & Aspectos de la apariencia física. \\
Clase 4 & Aspectos de la apariencia física. \\
Clase 5 & Compromiso y disposición para la atención. \\
\hline
\end{tabular}

Fuente: Elaboración propia de los autores (2019)

Con base a sus conectores se observa que para las clases $4-3$ y $1-5$ expresan cercanía y dependencia entre ambas, mientras que la clase 2 se torna independiente pero anidada a todas en conjunto, donde los criterios que añaden valor según son: elementos tangibles, fiabilidad, capacidad de respuesta, seguridad, y empatía. En la Tabla 2 se muestran los términos mencionados con mayor frecuencia, donde las palabras de la clase 4 y 3 hacen referencia a la dimensión de elementos tangibles, como: organización, estado, trabajador, verde -que hace referencia a las áreas verdes, biblioteca, hora, máquina, libro, señal, cobertura, entre otras que hacen mención a lo visible, mientras que la clase 1 y 5 hacen referencia a: empatía, fiabilidad y capacidad de respuesta, usando términos como tomar, cuenta, sistema, estudiante, baños, docente, prestar, opinión, personal, escuchar, brindaran, autoridad, comprometer, administrativo, respuesta.

Por último, la clase 2, en su rango muestra claramente el término seguridad, que prima en la percepción de las y los estudiantes, necesidad que los identifica para realizar sus estudios universitarios, lo que sustenta su sentir y pensar con las palabras más frecuentes como conocimiento $(\chi 2=192.6, \mathrm{p}<0.0001)$ y comodidad $(\chi 2=135.05$, $\mathrm{p}<0.0001)$, que lo observamos en la Tabla 3.

Tabla 3. Estadísticas de la percepción estudiantil de la calidad del servicio universitario en UNEMI vinculado a las clases.

\begin{tabular}{lllll}
\hline Clase 1 & Palabra & Tipo & Chi 2 & P \\
\hline 1 & Tomar & Verbo & 49.69 & 0.0001 \\
& Cuenta & Nombre & 34.44 & 0.0001 \\
& Sistema & Nombre & 22.9 & 0.0001 \\
& Estudiante & Nombre & 22.44 & 0.0001 \\
2 & Conocimiento & Nombre & 192.6 & 0.0001 \\
& Comodidad & Nombre & 135.05 & 0.0001 \\
& Seguridad & Nombre & 133.7 & 0.0001 \\
& Ayuda & Nombre & 126.34 & 0.0001 \\
3 & Organización & no & 24.64 & 0.0001 \\
& & reconocido & & \\
& Estado & Nombre & 20.16 & 0.0001 \\
\hline
\end{tabular}




\section{AMAZÓNAS}

\begin{tabular}{lllll}
\hline & Verde & Adjetivo & 20.16 & 0.0001 \\
Trabajador & Nombre & 20.16 & 0.0001 \\
Hacer & Verbo & 62.29 & 0.0001 \\
Están & no & 30.58 & 0.0001 \\
& reconocido & & \\
& Biblioteca & Nombre & 26.08 & 0.0001 \\
Hora & Nombre & 21.71 & 0.0001 \\
Baños & no & 37.17 & 0.0001 \\
& reconocido & & \\
& Jabón & no & 28.24 & 0.0001 \\
& reconocido & & \\
Docente & Adjetivo & 28.11 & 0.0001 \\
& nombre & 24.14 & 0.0001 \\
\hline
\end{tabular}

Fuente: Elaboración propia de los autores (2019).

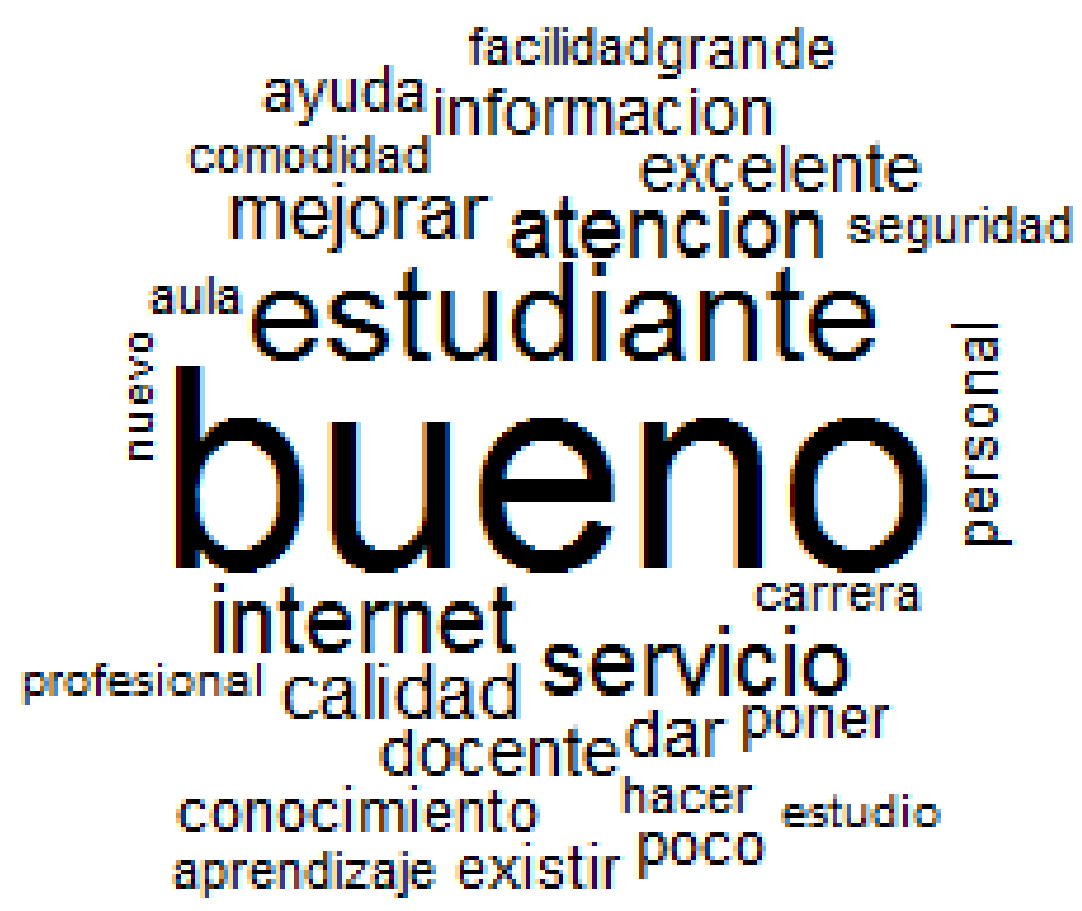

Figura 4. Nube de palabras de la percepción estudiantil de la calidad del servicio en UNEMI.

Fuente: Elaboración propia de los autores (2019).

En la Figura 4, muestra la agrupación y organización de palabras en función de su frecuencia, que resulta del análisis lexical, y califica la satisfacción del servicio como "bueno", según uso de palabra más frecuente, así también, incurren criterios percibidos que demandan la atención en criterios como: internet, docente, conocimiento, aprendizaje, atención, seguridad, información, facilidad, comodidad, personal, internet, aprendizaje, entre otros, que reafirman la escala dada bajo este criterio de análisis. 


\section{AMAZÓNAS}

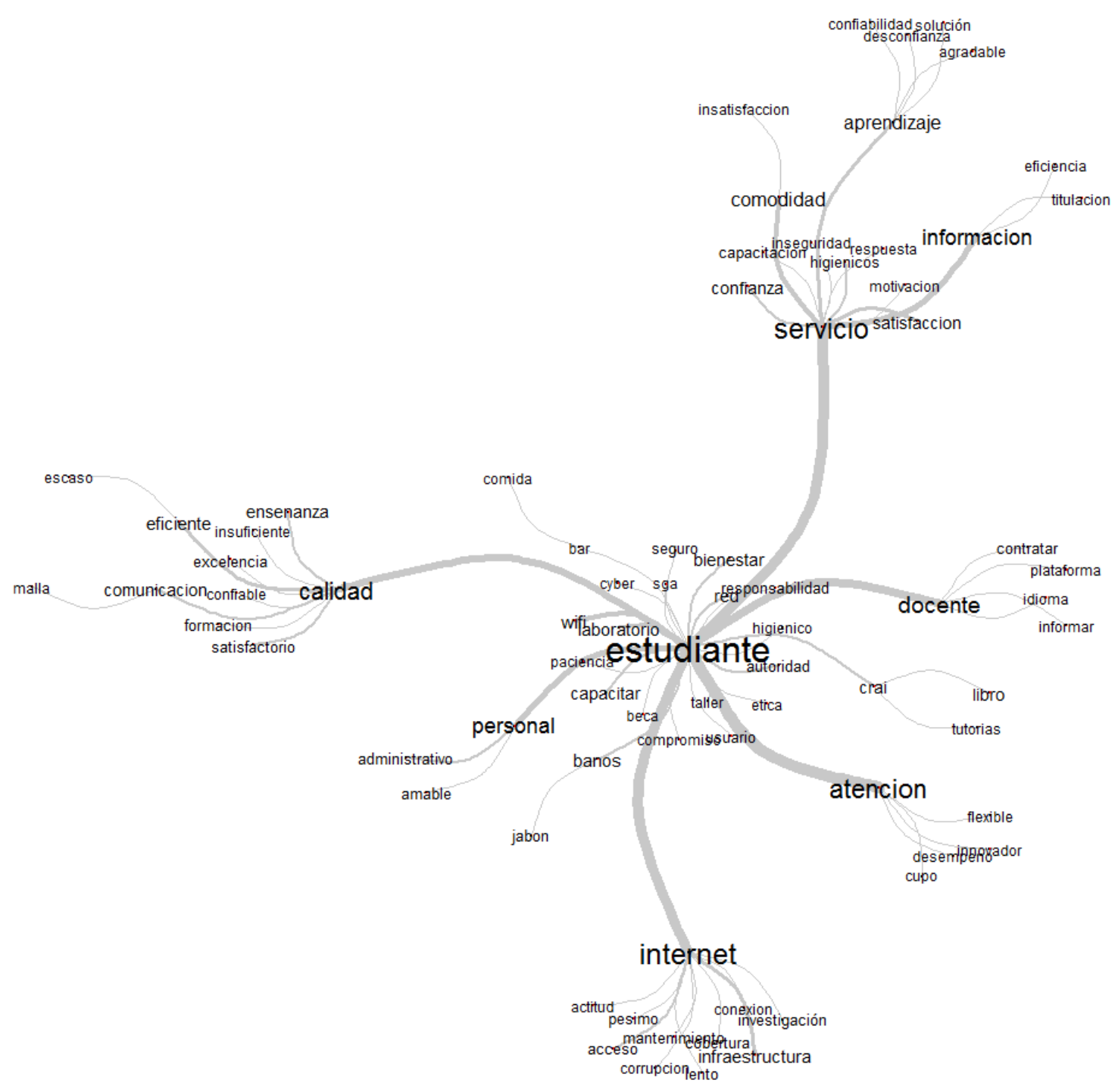

Figura 5. Análisis de similitud sobre la percepción estudiantil de la calidad del servicio en UNEMI.

Fuente: Elaboración propia de los autores (2019).

En la Figura 5, se evidencia que la calidad del servicio universitario bajo el conteo de concurrencias de palabras dadas en la encuesta por los estudiantes de UNEMI, en los términos más utilizados se relacionan con la necesidad del estudiante para sentirse cómodo y adaptado en el espacio universitario, centrada en el estudiante, y conexa al servicio y a su constructo teórico de calidad institucional, así como a términos específicos como la atención, el docente, el personal y el internet, que incide en las dimensiones del modelo Parasuraman.

\section{Resultados y discusión}

Resulta oportuno manifestar que el servicio universitario es responsabilidad y compromiso de todos sus actores, sin embargo, es el nivel estratégico de la institución el llamado a tomar decisiones y acciones oportunas para trabajar en el desarrollo de una 


\section{AMAZUNAS}

cultura de servicio, mediante políticas específicas que motiven a un servicio de excelencia, para minimizar la brecha que manifiestan algunos estudiantes entre el servicio esperado y el servicio percibido.

Por consiguiente, es importante la actuación responsable de la alta dirección en los criterios según los resultados obtenidos, desde la atención a los elementos tangibles, mediante la mejora de infraestructura, internet y espacios o áreas verdes, tomando en cuenta principalmente los talleres, laboratorios o centros de recursos de aprendizaje e investigación, como criterios demandantes en los estudiantes según los resultados encontrados, y que transmiten comodidad y satisfacción; en la capacidad de respuesta, fiabilidad y empatía, para que el servicio sea eficiente, y con este propósito trabajar en implementar un plan de perfeccionamiento de la competencia de orientación al servicio; continuando con el mismo orden y dirección, todas estas acciones serán base para coconstruir el elemento de seguridad en la comunidad universitaria sobre el trabajo realizado en pro de la comodidad y el conocimiento. Es necesario reflexionar que, de no tomarse las medidas correctivas sobre las percepciones del servicio universitario, la población estudiantil puede elegir o migrar a otras IES.

\section{Conclusiones}

Con base al análisis realizado y resultados obtenidos, se concluye que los términos léxicos señalados por los estudiantes, comodidad y conocimiento, tienen afinidad con las dimensiones de servicio del modelo de Parasuraman, que se enmarcan en el contexto de la gestión académica y administrativa de las IES.

En conclusión, los criterios mencionados por los estudiantes en el presente estudio, permiten conocer el nivel de calidad de las IES, por lo que es necesario que exista una evaluación interna y externa permanente con instrumentos técnicos especializados; que posteriormente permitan el análisis de resultados de forma crítica y reflexiva, y en efecto se propongan los debidos planes de acción para innovar las particularidades observadas en los servicios universitarios ofertados.

\section{Referentes Bibliográficas}

Aguilar-Gordón, F. del R. (2018). Fundamento, evolución, nodos críticos y desafíos de la educación ecuatoriana actual. Actualidades Investigativas En Educación, 19(1). https://doi.org/10.15517/aie.v19i1.35715

Alvarado-Lagunas, E., Luyando-Cuevas, J. R., \& Picazzo-Palencia, E. (2015). Percepción de los estudiantes sobre la calidad de las universidades privadas en Monterrey. Revista Iberoamericana de Educación Superior, 6(17), 58-76. https://doi.org/10.22201/iisue.20072872e.2015.17.162

Alvarado Peña, L. J., \& Díaz De León, F. I. (2015). Calidad del servicio en universidades públicas mexicanas: perspectiva de análisis desde las dimensiones. Quality of the Service in Public Mexican Universities: Perspective of Analysis from the Dimensions., (38), 95-118. Retrieved from http://search.ebscohost.com/login.aspx?direct=true\&db=asn\&AN=108525289\&lang $=\mathrm{es}$

Asamblea Nacional del Ecuador. (2010). Ley Orgánica De Educación Superior Ecuador. 
Registro Oficial Suplemento 298 de 12-Oct.-2010, 92. Retrieved from http://educaciondecalidad.ec/leyes-sistema/ley-educacion-superior-loes.html

Carrasco Proaño, Angelica; Cuzco Lopez, Miriam; Correa Peralta, Mirella; Vinueza Martínez, Jorge; Cabrera Torres, A. (2018). Análisis de planificación estratégica para las actividades artesanales : SCOPUS 2014 a 2016.

Carretero Diaz, M. A. (2004). La calidad: a la búsqueda de la confianza perdida. Una reflexión crítica sobre la calidad y su reflejo en el entorno educativo. Revista Complutense de Educación, 15(2), 521-560.

Cervantes, V., Peralta, P., Salgado, R., \& Stefanell, I. (2018). Calidad De Servicio En Una Institución De Educación Superior En La Ciudad De Barranquilla. Ciencias Administrativas, $\quad 11(6), \quad 28-40 . \quad$ Retrieved from http://revistas.unlp.edu.ar/CADM\%0ACALIDAD

Consejo de Evaluación, acreditación y aseguramiento de la calidad de la E. S. (2018). Ceaaces, 1-26.

Correa, V., Agila, G., José, J., \& Palacios, O. (2012). Sensación y percepción en la construcción del conocimiento. Sophia, Colección de Filosofía de La Educación, 13(13), 123-149. Retrieved from https://books.google.com.ec/books?id=iliU7qcQy4oC\&pg=PA257\&lpg=PA257\&dq =la+percepción+es+una+competencia+especial+de+la+conciencia,+se+trata+incluso $+\mathrm{de}+$ su+capacidad+por+antonomasia" \&source=bl\&ots=v7xMbEA9oA\&sig=ACfU3 U0vDznmyklH36T38z91DfJOQGg\%0Ahttp://bvi

D’Armas Regnault, M., Robles Salguero, R., Cedillo Fajardo, M., Vinueza Martínez, J., Correa Peralta, M., Mejías Acosta, A., \& Acosta Nuñez, G. (2018). Imagen institucional universitaria: Un estudio de caso en el contexto del Ecuador. Espacios, $39(5)$.

Duque Oliva, E. J. (2005). Revisión del concepto de calidad del servicio y sus modelos de medición *.

Duque Oliva, E. J., \& Chaparro Pinzón, C. R. (2017). Medición De La Percepción De La Calidad Del Servicio De Educación Por Parte De Los Estudiantes De La Uptc Duitama. Criterio Libre, 10(16), 159. https://doi.org/10.18041/19000642/criteriolibre.16.1168

Ecuador. (2008). Constitución del Ecuador. Registro Oficial, (20 de Octubre), 173. https://doi.org/10.1017/CBO9781107415324.004

Espinosa, M., Gutiérrez, C., Muñoz, R., \& Víctor, M. (2017). ESTUDIANTES: SUS PERCEPCIONES SOBRE LA CALIDAD EDUCATIVA Y SU IMPORTANCIA PARA EL COMPROMISO SOCIAL UNIVERSITARIO.

Feldman, A. N. (2002). LA INTEGRACIÓN DEL ANÁLISIS TEXTUAL A LOS ESTUDIOS DE CALIDAD DE SERVICIO Y SATISFACCIÓN DEL CLIENTE, (1994), 265-276.

Iacovidou, M., Gibbs, P., \& Zopiatis, A. (2009). Quality in Higher Education An Exploratory Use of the Stakeholder Approach to Defining and Measuring Quality: The Case of a Cypriot Higher Education Institution, (December 2014), 37-41. https://doi.org/10.1080/13538320902995774

Ortega Carrasco, R. J., Veloso Toledo, R. D., \& Hansen, O. S. (2018). Percepción y actitudes hacia la investigación científica. Academo Revista de Investigación En Ciencias Sociales y Humanidades, 5(2), 101-109. https://doi.org/10.30545/academo.2018.jul-dic.2

Ortega Márquez, M., \& Márquez Fernández, O. (2017). Percepción social del servicio de 


\section{AMAZÉNAS}

agua potable en el municipio de Xalapa, Veracruz. Revista Mexicana de Opinión Pública, 23(23), 41. https://doi.org/10.22201/fcpys.24484911e.2017.23.58515

Palominos, P., Quezada, L., Osorio, C., Torres, J., \& Lippi, L. (2016). Calidad de los servicios educativos según los estudiantes de una universidad pública en Chile. Revista Iberoamericana de Educación Superior, 7(18), 130-142. Retrieved from https://ries.universia.net/article/view/1132/1775

Rincón Finol, Imelda; Ortega Moreno, Sheila (2018). Vigencia de la reforma universitaria de Córdoba en la gestión pública. Revista de la Universidad del Zulia. Volumen 9, Número 25, Septiembre-Diciembre 2018, 98-103. Maracaibo: Universidad del Zulia. www.produccioncientificaluz.org/index.php/rluz/article/view/29746

Roccaro, I. E. (2002). El paradigma de calidad reformada en el servicio público universitario, 33-34.

Rojo, G. (2017). 1. Introducción.

Romero-Pérez, I., Alarcón-Vásquez, Y., \& García-Jimenez, R. (2018). Lexicometría: enfoque aplicado a la redefinición de conceptos e identificación de unidades temáticas. Biblios: Journal of Librarianship and Information Science, 71(71), 68-80. https://doi.org/10.5195/biblios.2018.466

Salazar Yépez, W., \& Cabrera Vallejo, M. (2016). Diagnóstico de la calidad de servicio, en la atención al cliente, en la Universidad Nacional de Chimborazo- Ecuador. Industrial Data, 19(2), 13. https://doi.org/10.15381/idata.v19i2.12811

Saltos Aguilar, W. M., \& Arguello Erazo, S. E. (2017). La calidad de los servicios que perciben los estudiantes de la Universidad Nacional de Chimborazo de la ciudad de $\begin{array}{lllll}\text { Riobamba - } & \text { Ecuador. Industrial }\end{array}$ https://doi.org/10.15381/idata.v20i1.13517

Suescún Guerrero, W. D. (2015). El discurso educativo en profesores universitarios bajo la percepción de sus estudiantes. Lengua y Habla, (19), 267-296.

Tumino, M., \& Poitevin, E. (2013). Evaluación de la calidad de servicio universitario desde la percepción de estudiantes y docentes: caso de estudio. REICE. Revista Iberoamericana Sobre Calidad, Eficacia y Cambio En Educación, 12(2), 63-84. Retrieved from https://revistas.uam.es/index.php/reice/article/view/2856/3073

Vergara, J. C. y Quesada, V. M. (2011). (2011). Análisis de la calidad en el servicio y satisfacción de los estudiantes de Ciencias Económicas de la Universidad de Cartagena mediante un modelo de ecuaciones estructurales, 13, 108-122.

Villalba Sanchez, C. (2013). La calidad del servicio : un recorrido histórico conceptual, sus modelos más representativos y su aplicación en las universidades. 